\title{
Synthetic Aperture Radiometry Evaluated by a Two-Channel Demonstration Model
}

\author{
Laursen, Brian; Skou, Niels
}

Published in:

I E E E Transactions on Geoscience and Remote Sensing

Link to article, DOI:

$10.1109 / 36.673675$

Publication date:

1998

Document Version

Publisher's PDF, also known as Version of record

Link back to DTU Orbit

Citation (APA):

Laursen, B., \& Skou, N. (1998). Synthetic Aperture Radiometry Evaluated by a Two-Channel Demonstration Model. I E E E Transactions on Geoscience and Remote Sensing, 36(3), 822-832.

https://doi.org/10.1109/36.673675

\section{General rights}

Copyright and moral rights for the publications made accessible in the public portal are retained by the authors and/or other copyright owners and it is a condition of accessing publications that users recognise and abide by the legal requirements associated with these rights.

- Users may download and print one copy of any publication from the public portal for the purpose of private study or research.

- You may not further distribute the material or use it for any profit-making activity or commercial gain

- You may freely distribute the URL identifying the publication in the public portal

If you believe that this document breaches copyright please contact us providing details, and we will remove access to the work immediately and investigate your claim. 


\title{
Synthetic Aperture Radiometry Evaluated by a Two-Channel Demonstration Model
}

\author{
Brian Laursen, Member, IEEE, and Niels Skou, Senior Member, IEEE
}

\begin{abstract}
The Technical University of Denmark (TUD) Synthetic Aperture Radiometer (SARad) is a two-channel demonstration model that can simulate a two-dimensional (2-D) thinned array radiometer having an unfilled aperture populated with several small antenna elements. Aperture synthesis obtained by interferometric measurements using the antenna elements in pairs, followed by an image reconstruction based on an inverse Fourier transform, results in an imaging instrument without the need of mechanical scan. The thinned aperture and the nonscanning feature make the technique attractive for spaceborne radiometer systems, especially at low frequencies.

The TUD SARad demonstration model consists of a twochannel $K_{u}$-band correlation radiometer with two horn antennas and an antenna mounting structure enabling the horns to be mounted in relevant positions within a certain aperture. A total aperture synthesis is obtained by sequentially placing the two antenna elements in all required pairs of positions and measuring the corresponding samples of the visibility function.

The system has been used to demonstrate 2-D synthetic aperture imaging of complex targets in outdoor ground experiments. A special feature of the system is that it uses a focused antenna system, thus enabling a short distance to the target, yet still utilizing image reconstruction algorithms identical to those used in a normal far-field situation.

The aperture synthesis theory is discussed, with special emphasis on focused systems; the radiometer system is described; and images suitable for demonstration of resolution and other imaging properties are presented and discussed.
\end{abstract}

Index Terms-Aperture synthesis, radiometer.

\section{INTRODUCTION}

$\mathbf{T}$ HE SYNTHETIC Aperture Radiometer (SARad) systems are fundamentally different from traditional radiometer systems in that they measure in the spatial frequency domain and an inverse Fourier transformation is needed to obtain the normal brightness temperature distribution in the spatial domain. Aperture synthesis involves interferometric measurements made by cross correlating the signals from two spatially separated antennas having overlapping field of view.

The cross correlation of the signals from the two antennas (having passed through two properly designed radiometers) produces a sample of the visibility function corresponding to the relative positions of the antenna elements (the visibility function is the Fourier transform of the brightness temperature distribution within the image). By making a new measurement

Manuscript received July 1, 1996; revised September 18, 1997. This work was supported by the European Space Agency and the Danish National Research Foundation.

The authors are with the Danish Center for Remote Sensing, Department of Electromagnetic Systems, Technical University of Denmark, 2800 Lyngby, Denmark (e-mail: bl@emi.dtu.dk).

Publisher Item Identifier S 0196-2892(98)01138-3. with another relative position of the antennas, another sample of the visibility function is found. Only a limited number of measurements, i.e., positions, are required to adequately sample the visibility function. In a full-fledged imaging system, this is done by having a suitable number of (small) antenna elements placed properly within the aperture and then simultaneously cross correlate all possible pairs to generate the visibility function.

The one-by-one measurement of the visibility samples in the demonstration model requires the test scene and the electronics to remain stable for a considerable time-typically a few hours. Temperature regulation ensuring long-term stability of the radiometers is needed, and calibration must be checked frequently. An IF switch arrangement in front of the analog complex correlator enables the cross correlator to operate as two self-correlators (identical to detectors) and hereby calibrate the two radiometers as well as the correlators in a traditional way. Offsets on the output of the correlators are reduced by phase switching the local oscillator signals and properly demodulating after the correlators.

The sampling distance (or the minimum antenna spacing) is chosen so that all visibility samples are measured. The antenna elements have an outer diameter slightly larger than $1.5 \lambda$. The sampling distance is $1.57 \lambda$, and the maximum aperture is $32 \lambda$, resulting in a synthetic beamwidth of $2.2^{\circ}$ at nadir and an image size of $18 \times 18$ pixels. To enable a practical distance between the aperture and the target, the system is focused by having the antenna elements mounted on a curved rail. By rotating this rail around a vertical axis, the full aperture can be covered.

The purpose of such a two-channel demonstration model is twofold: with relatively cheap instrumentation (only two channels), the SARad concept can be demonstrated and investigated, and specific configurations for airborne or spaceborne systems can be simulated and evaluated while they are still in the design phase.

\section{SARad THEORY}

The general theory concerning radiometry using interferometric aperture synthesis is published in [1] and [2].

Let the visibility function be the Fourier transform of the variation in brightness temperature within the image. Samples of the visibility function $V\left(\boldsymbol{a}_{1}, \boldsymbol{a}_{2}\right)$ are found as the complex cross correlation between signals (voltages $v_{1}(t)$ and $v_{2}(t)$ ) from two antennas

$$
V\left(\boldsymbol{a}_{1}, \boldsymbol{a}_{2}\right)=\left\langle v_{1\left(0^{\circ}\right)}(t) v_{2\left(0^{\circ}\right)}(t)\right\rangle+j\left\langle v_{1\left(-90^{\circ}\right)}(t) v_{2\left(0^{\circ}\right)}(t)\right\rangle .
$$


Let the position of the antennas be given by the vectors $\boldsymbol{a}_{1}=\left(x_{A 1}, y_{A 1}, z_{A 1}\right)$ and $\boldsymbol{a}_{2}=\left(x_{A 2}, y_{A 2}, z_{A 2}\right)$, in accordance with Fig. 1, and the visibility function is given by [2]

$$
\begin{aligned}
V\left(\boldsymbol{a}_{1}, \boldsymbol{a}_{2}\right)= & \int_{0}^{2 \pi} \int_{0}^{\pi} \frac{T_{B}(\theta, \phi)}{4 \pi} G(\theta, \phi) r(\Delta \mathrm{R} / c) e^{j \frac{2 \pi}{\lambda} \Delta R} \\
& \times \sin (\theta) d \theta d \phi
\end{aligned}
$$

where

$T_{B}(\theta, \phi)$ brightness temperature from a point in direction $(\theta, \phi)$

$G(\theta, \phi)$ antenna element gain;

$r(\Delta \mathrm{R} / c) F^{-1}\left\{H_{1}\left(f-f_{C}\right) H_{2}^{*}\left(f-f_{C}\right)\right\}$;

$f_{C}$ center frequency;

$\Delta R$ difference of distance from the two antennas to a point in direction $(\theta, \phi)$, i.e. $\Delta R=R_{2}-R_{1}$;

$\lambda$ wavelength.

$r(\Delta R / c)$ is a function that describes the effect on the correlation when the difference in time between the signals is large. Looking at the correlation between signals from a direction far away from the center and at the same time measuring a visibility sample with a large distance between the two antennas, the large time difference between the signals will result in a decreasing correlation as a function of the system bandwidth. $H_{1}(f)$ and $H_{2}(f)$ are the system filter response of the two particular channels. In case of a rectangular filter response, we have $r(\Delta R / c)=\operatorname{sinc}\left(\pi B_{\text {Sys }} \Delta \mathrm{R} / c\right)$, where $B_{\text {Sys }}$ is the system bandwidth.

\section{A. Near-Field Geometry}

In the following, it will be assumed that signals from behind the aperture can be neglected, and a subscript ()$_{m}$ indicates signals from the half space in front of the aperture (signals from behind the aperture cannot be distinguished from signals from a direction in front of the aperture). It is also assumed that the system is nadir looking on a flat ground $z_{G}=h$, where the $(x, y, z)$ and the spherical $(r, \theta, \phi)$ coordinate systems are given in Fig. 1.

The distance from the two antennas to a point $\left(x_{G}, y_{G}, z_{G}=\right.$ $h$ ) denoted, respectively, as $R_{1}$ and $R_{2}$ are given by

$$
\begin{aligned}
& R_{1}=\sqrt{\left(x_{G}-x_{A 1}\right)^{2}+\left(y_{G}-y_{A 1}\right)^{2}+\left(h-z_{A 1}\right)^{2}} \\
& R_{2}=\sqrt{\left(x_{G}-x_{A 2}\right)^{2}+\left(y_{G}-y_{A 2}\right)^{2}+\left(h-z_{A 2}\right)^{2}} .
\end{aligned}
$$

Placing the antennas on a spherical surface with the center of the sphere at the center of the scene (radius of sphere equal to $h$ ) and, by introducing $r_{G}=\sqrt{x_{G}^{2}+y_{G}^{2}+h^{2}}$, (3) and (4) can be written as

$$
\begin{aligned}
R_{1}=r_{G}\left[1+\frac{(-1)}{2 r_{G}^{2}}\left(2 x_{G} x_{A 1}+2 y_{G} y_{A 1}\right)\right. \\
\left.-\frac{(-1)^{2}}{8 r_{G}^{4}}\left(2 x_{G} x_{A 1}+2 y_{G} y_{A 1}\right)^{2}+\sigma_{1}\right] \\
R_{2}=r_{G}\left[1+\frac{(-1)}{2 r_{G}^{2}}\left(2 x_{G} x_{A 2}+2 y_{G} y_{A 2}\right)\right. \\
\left.-\frac{(-1)^{2}}{8 r_{G}^{4}}\left(2 x_{G} x_{A 2}+2 y_{G} y_{A 2}\right)^{2}+\sigma_{2}\right]
\end{aligned}
$$

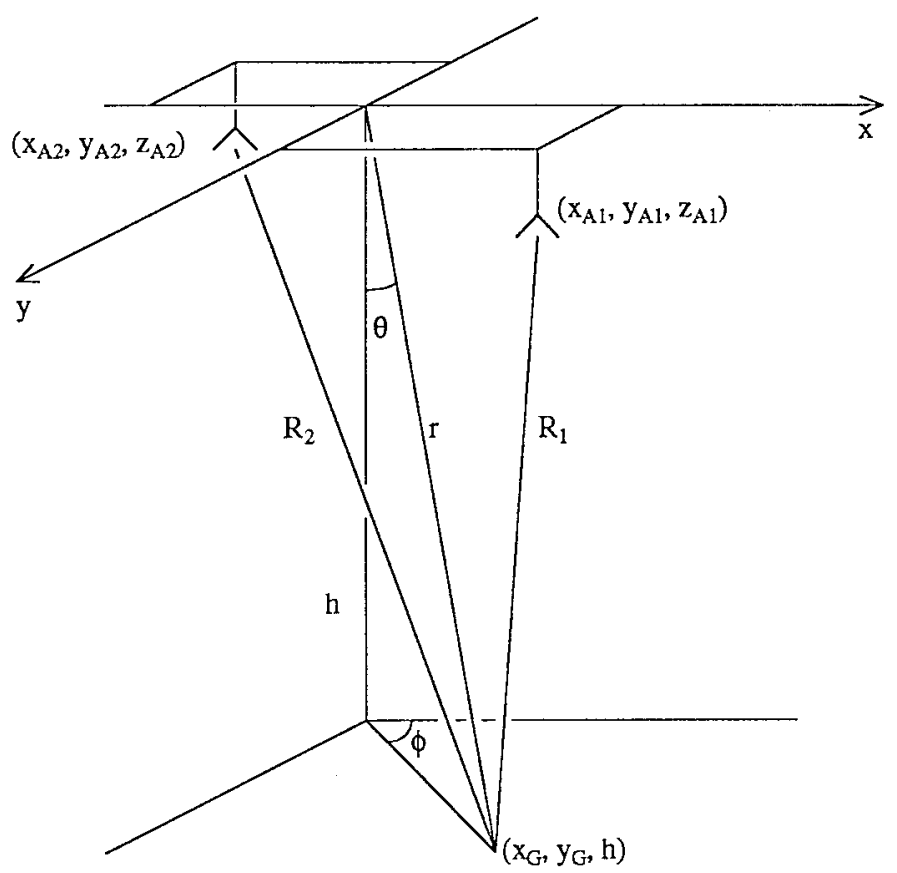

Fig. 1. Two-dimensional nadir-looking synthetic aperture interferometer.

where $\sigma$ is the remainder after a second-order Taylor approximation. If the antennas are placed symmetrically about the origin of the $x, y$ plane, $x_{A 2}=-x_{A 1}$ and $y_{A 2}=-y_{A 1}$, the second-order term, and all other terms of even order cancel when the difference of distances from a ground pixel to the two antennas is determined

$$
\begin{aligned}
\Delta R & =R_{2}-R_{1} \\
& \approx 2 x_{A 1} \frac{x_{G}}{\sqrt{x_{G}^{2}+y_{G}^{2}+h^{2}}}+2 y_{A 1} \frac{y_{G}}{\sqrt{x_{G}^{2}+y_{G}^{2}+h^{2}}} .
\end{aligned}
$$

Introducing the spherical coordinate system $(r, \theta, \phi)$ and defining the spatial frequencies $(u, v)$ as the distance between the antenna elements in $x$-axis and $y$-axis directions, measured in wavelength $u=\left(x_{A 1}-x_{A 2}\right) / \lambda=2 x_{A 1} / \lambda$ and $v=$ $\left(y_{A 1}-y_{A 2}\right) / \lambda=2 y_{A 1} / \lambda$, (7) can be written as

$$
\frac{\Delta R}{\lambda} \approx u \sin (\theta) \cos (\phi)+v \sin (\theta) \sin (\phi) .
$$

The same equation is achieved in the case of an object placed in the far field of the synthetic aperture, but without the restriction that the synthetic aperture should be a spherical surface and that the antenna pairs should be placed symmetrically. The separation criterion between near field (aperture is a spherical surface) and far field (flat aperture) is

$$
R_{\text {far field }}>20 \frac{D_{\mathrm{SYN}}^{2}}{\lambda}
$$

where

$D_{\mathrm{SYN}}$ diameter (size) of the synthetic aperture.

Thus, in general, airborne and spaceborne systems use a flat aperture, while compact ground-based setups, with the target in the near field of the synthetic aperture, must use a focused approach if we want to use identical algorithms for image reconstruction. 


\section{B. Sampling of the Visibility Function}

The following considerations are valid for both near-field and far-field systems. The sampling conditions and the normalization of the measured visibility function are discussed.

The relationship between the measured visibility function and the brightness temperature is given (10), where the coupling between the antenna elements is neglected (this assumption is fulfilled in the TUD demonstration model, see Section IV)

$$
\begin{aligned}
V(u, v)= & \int_{0}^{2 \pi} \int_{0}^{\pi} \frac{T_{B}(\theta, \phi)}{4 \pi} G(\theta, \phi) r(\Delta R / c) \\
& \times e^{j 2 \pi(u \sin (\theta) \cos (\phi)+v \sin (\theta) \sin (\phi))} \sin (\theta) d \theta d \phi .
\end{aligned}
$$

Recalling that only signals from directions in front of the aperture are considered, the $(\xi, \eta)$ coordinates can be defined as

$$
\begin{aligned}
& \xi=\sin (\theta) \cos (\phi) \\
& \eta=\sin (\theta) \sin (\phi) .
\end{aligned}
$$

Let us choose a system bandwidth $B_{\text {sys }}$, so that the decorrelation effect can be neglected $(r(\Delta R / c) \approx 1)$. In our case (see Section IV), measurements are taken outdoors and the combination of low brightness temperature in the back lobe (the sky brightness temperature of a few Kelvin) and a relatively low antenna gain in the back lobe allows the signals from directions behind the aperture to be neglected. The visibility function is then written as

$$
\begin{aligned}
V(u, v)= & \iint_{\xi^{2}+\eta^{2}<1} \frac{T_{B, m}(\xi, \eta)}{4 \pi} G_{m}(\xi, \eta) \\
& \times \frac{1}{\sqrt{1-\xi^{2}-\eta^{2}}} e^{j 2 \pi(u \xi+v \eta)} d \xi d \eta .
\end{aligned}
$$

It is seen, that the complex cross correlation between signals from two antennas is a simple Fourier sampling operator in the spatial frequencies $(u, v)$. The exponential function with the complex argument determines the frequency. The sampling distance is in general chosen to be $d u=d v=0.5$, but the sampling distance can often be increased to reduce the number of receivers for a given synthetic aperture size [3]. The relation between the size of the reconstructed image $(\Delta \xi$ and $\Delta \eta)$ and the spatial sampling distance $(d u$ and $d v)$ is

$$
\Delta \xi=\frac{1}{d u} \quad \text { and } \quad \Delta \eta=\frac{1}{d v}
$$

where $\Delta \xi$ is the swath.

Let the area that covers the reconstructed image $D_{0}$ be defined as

$$
D_{0}:(\xi, \eta) \in\left(\left[\frac{-\Delta \xi}{2}, \frac{\Delta \xi}{2}\left[,\left[\frac{-\Delta \eta}{2}, \frac{\Delta \eta}{2}[)\right.\right.\right.\right.
$$

and the visibility function is given by

$$
\begin{aligned}
V(u, v)= & \iint_{(\xi, \eta) \in D_{0}} \frac{T_{B, m}(\xi, \eta)}{4 \pi} G_{m}(\xi, \eta) \\
& \times \frac{1}{\sqrt{1-\xi^{2}-\eta^{2}}} e^{j 2 \pi(u \xi+v \eta)} d \xi d \eta \\
& +\iint_{(\xi, \eta) \notin D_{0}} \frac{T_{B, m}(\xi, \eta)}{4 \pi} G_{m}(\xi, \eta) \\
& \times \frac{1}{\sqrt{1-\xi^{2}-\eta^{2}}} e^{j 2 \pi(u \xi+v \eta)} d \xi d \eta .
\end{aligned}
$$

To be able to reconstruct the image properly without aliasing, it is a requirement that the signals from the area $(\xi, \eta) \notin D_{0}$ vanish. It is possible, however, to compensate for constant signals from the area $(\xi, \eta) \notin D_{0}$, like the sky background radiation. The elements have a rather narrow radiation pattern, yet not narrow enough to neglect signals from the area surrounding the scene. However, for the ground experiments presented in the following, the radiation from the relevant parts of this area is known (area covered by microwave absorbers of known temperature) and compensated for in the image processing [see (17)].

Let $V^{\prime}(u, v)$ be defined as

$$
\begin{aligned}
V^{\prime}(u, v)= & \iint_{(\xi, \eta) \in D_{0}} T_{B, m}(\xi, \eta) \frac{G_{m}(\xi, \eta)}{4 \pi} \\
& \times \frac{1}{\sqrt{1-\xi^{2}-\eta^{2}}} e^{j 2 \pi(u \xi+v \eta)} d \xi d \eta .
\end{aligned}
$$

$V(u, v)$ is what is measured by the instrument, while $V^{\prime}(u, v)$ is the calibrated visibility function to be used in the image reconstruction $\left(V^{\prime}(u, v)\right.$ is the frequency components from the area defined by $\left.D_{0}\right) . V^{\prime}(u, v)$ is found by combining (15) and (16) as

$$
\begin{array}{r}
V^{\prime}(u, v)=\left(V(v, u)-\iint_{(\xi, \eta) \notin D_{0}} \frac{T_{B, m}(\xi, \eta)}{4 \pi} G_{m}(\xi, \eta)\right. \\
\left.\quad \times \frac{1}{\sqrt{1-\xi^{2}-\eta^{2}}} e^{j 2 \pi(u \xi+v \eta)} d \xi d \eta\right)
\end{array}
$$

where $T_{B, m}(\xi, \eta)$ is the physical temperature of the absorbers. Let the visibility function be decomposed as

$$
V_{e}^{\prime}(u, v)=V^{\prime}(u, v)-T_{s}^{\prime} \frac{\hat{G}_{m}(u, v)}{4 \pi}
$$

where

$$
\begin{array}{ll}
T_{s}^{\prime} & V^{\prime}(0,0) ; \\
\hat{G}_{m}(u, v) & \begin{array}{l}
\text { Fourier transform of the antenna gain on the } \\
\text { measured }(u, v) \text { grid. }
\end{array}
\end{array}
$$


The image reconstruction can now be based on an inverse Fourier transform $F^{-1}$ of $V_{e}^{\prime}(u, v)$ :

$$
T_{B}(\xi, \eta)=\frac{4 \pi}{G_{m}(\xi, \eta)} \sqrt{1-\xi^{2}-\eta^{2}} F^{-1}\left\{V_{e}^{\prime}(u, v)\right\}+T_{s}^{\prime}
$$

\section{CALiBration}

Instrument biases in total-power radiometers are basically caused by gain and noise figure instability. The biases are more complicated in correlation radiometers, where offset on the correlation output can be very critical, as the correlation often is less than $1 \%$.

The offsets in the correlator outputs mainly result from imperfections in circuits or from a correlation between spurious signals.

Offset currents in the analog correlator (or drifts in the quantization levels in a digital correlator) will give rise to offsets in the correlator outputs. Cross coupling of small amounts of noise from one receiver to another will give rise to offsets as well. Since all visibility samples are measured with the same two receivers and since cross coupling between antenna elements can be neglected in the present case (see Section IV), offsets on the visibility samples tend to be the same for all samples. Such errors will show up as spurious signals at the center of the reconstructed image.

Offsets are difficult to eliminate entirely without phase switching. Phase switching is, in the simple case, a $180^{\circ}$ phase switching of the local oscillator signals and proper demodulation after the correlator. If the duty cycle is exactly $50 \%$, offsets are canceled.

Only spurious signals that enter the signal channels at a point that comes after the phase switch will be reduced by phase switching. The closer the phase switch is placed to the antenna, the more effective the result will be.

The real life efficiency of the phase switching to reduce the offsets, is around two or more orders of magnitude [1], dependent on the nature of the spurious signals.

The implementation of phase switching is very simple for the two channel correlation radiometer. A $180^{\circ}$ phase switching with $50 \%$ duty cycle of the local oscillator signal in one of the channels is incorporated in the RF (microwave) section (see Fig. 2).

The phase switching is performed at the local oscillator, rather than on the full signal band, to avoid the possibility of introducing distortion in the frequency response. The phase switching demodulation is placed after the analog correlator because offset currents in the multiplier are giving the most significant contribution to the offset.

\section{DEMONSTRATION MODEL}

The TUD $K_{u}$-band two-channel correlation radiometer employs two single-sideband superheterodyne receivers. The RF center frequency is $16.25 \mathrm{GHz}$ and the IF center frequency is $250 \mathrm{MHz}$ with a $100-\mathrm{MHz}$ system bandwidth.

The measurement setup consists of the correlation radiometer with two horn antennas and an antenna mounting structure

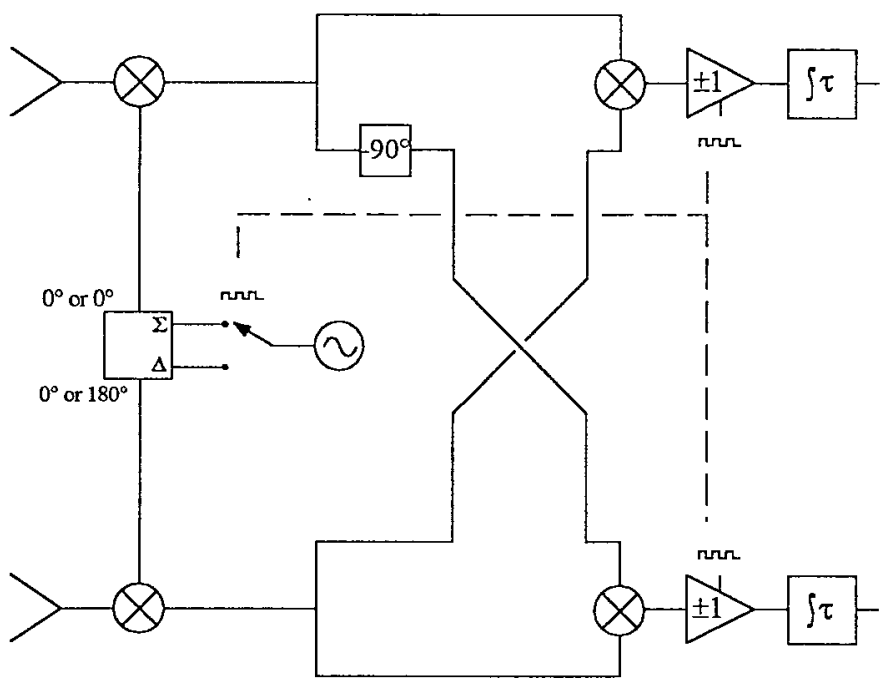

Fig. 2. Phase switching with analog correlators.

enabling the horns to be mounted in relevant positions within a certain aperture. A total aperture synthesis is obtained by sequentially placing the two antenna elements in all possible, required pairs of positions and measuring the corresponding samples of the visibility function. The demonstration model, thus, requires the test scene to remain stable for a considerable time-typically a few hours.

\section{A. Antenna Mounting Structure}

In the normal, focused configuration, the demonstration model is nadir looking. The experiments are carried out outdoors, and the scene consists of metal targets reflecting the sky (low brightness temperature) against a warm, uniform background. In one example to be discussed in Section VI, metal letters spelling "EMI" have been used as targets.

To enable a practical distance between the aperture and the target, the antenna elements are mounted on a curved rail [see Fig. 3(a)]. The horns are always mounted symmetrically about the center of the rail. It is shown in (8) that the curved rail concept in the near-field antenna system is equivalent to a "flat" antenna in the far-field case, with respect to interference patterns.

By rotation of the rail around a vertical axis, the full aperture can be covered. The measured spatial frequencies are shown in Fig. 3(b), given by the $(u, v)$ coordinates. A photo, taken looking upwards, of the antenna mounting structure is shown in Fig. 4.

The visibility function is sampled with a $1.57 \lambda$ spacing on the rail. During measurements, the rail is rotated in steps, such that the angular sampling distance corresponds to a $1.57 \lambda$ spacing between visibility samples on the largest circle in the $(u, v)$ plane [see Fig. 3(b)]. (The $(u, v)$ coordinates are the distances between the antenna elements measured in wavelengths.)

It may be mentioned here that the demonstration model has also been used in far-field experiments with a Y-shaped, flat antenna structure (see [4]). 


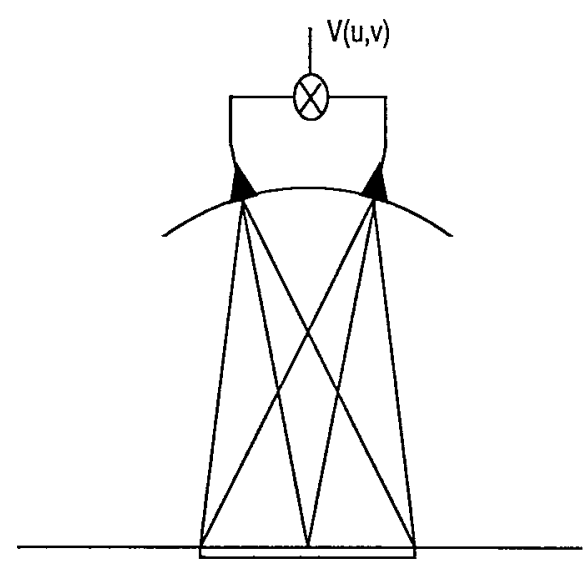

(a)

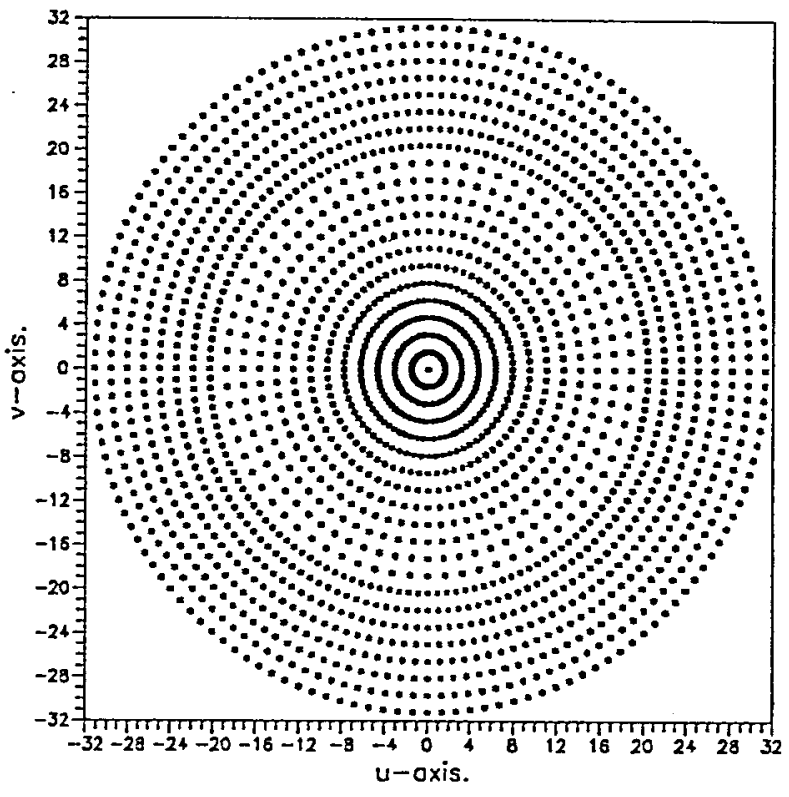

(b)

Fig. 3. (a) Focused antennas in the near-field synthetic aperture. The full aperture is covered by rotation of the curved rail around a vertical axis. (b) Measured spatial frequencies $(u, v)$ for the near-field setup.

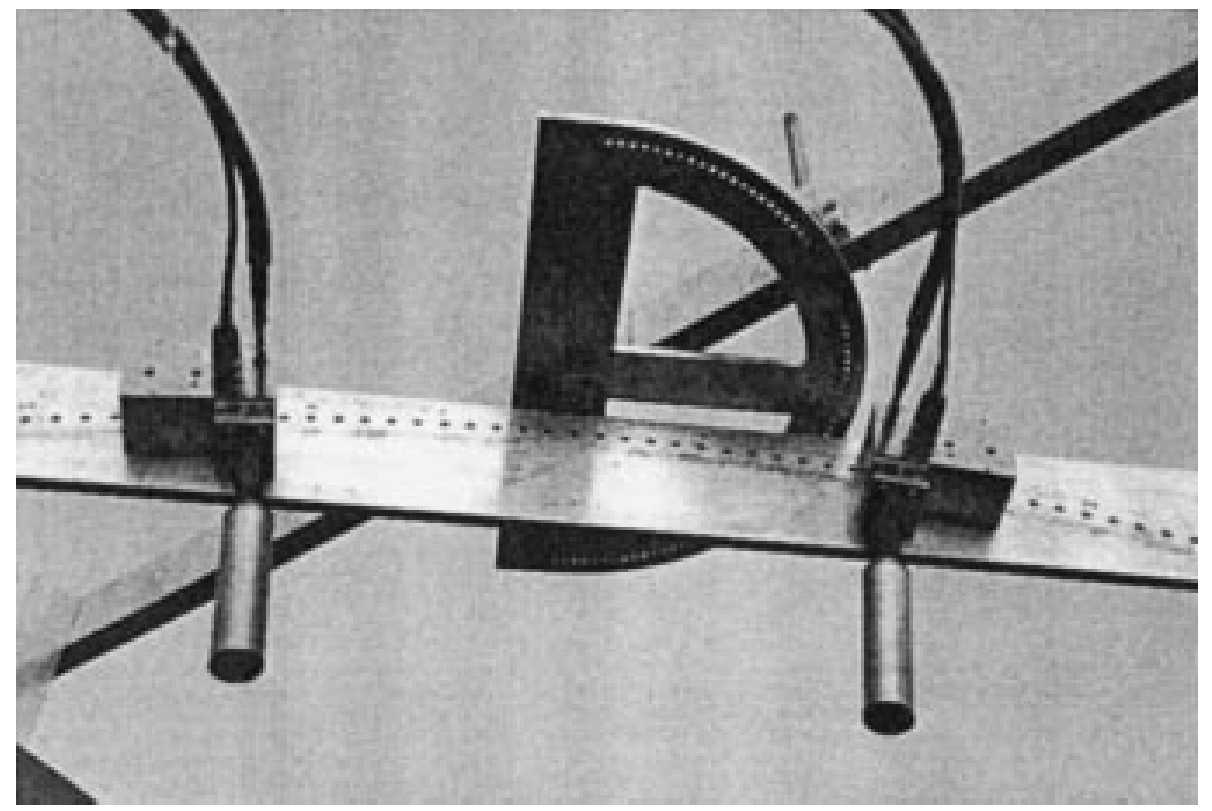

Fig. 4. Antenna element mounting structure (near field).

\section{B. Antenna Elements}

The basic image reconstruction is an inverse fast Fourier transform (FFT), followed by an antenna gain correction. In this case, all antenna patterns must be the same.

Small circular dual mode horns with a $1.5 \lambda$ diameter are used as antenna elements. Using a $1.57 \lambda$ distance between the antenna element positions in the near-field setup, all visibility samples (including the lowest frequency visibility samples) are measured. The reconstructed image is $\pm 19^{\circ}$ from nadir. The FFT algorithm can only be used provided there is no coupling between the antennas (see [5] and [6]). The coupling between the dual-mode circular horns is measured to be $-45 \mathrm{~dB}$ worst case and can be ignored. The rotation of the rail, and, herewith, a rotation of the antenna elements bring about an additional requirement to the antenna elements; identical radiation patterns in $E$ - and $H$-planes are required within the $\pm 19^{\circ}$ used for imaging. The dual-mode horns are optimized to obtain nearly identical radiation patterns in $E$ - and $H$ plane within the reconstructed field of view (see Fig. 5). The antenna patterns have rotation symmetry to within $0.12 \mathrm{~dB}$. Even this may correspond to an error of up to $4.2 \mathrm{~K}$, and stringent demonstration of calibration would thus require an 


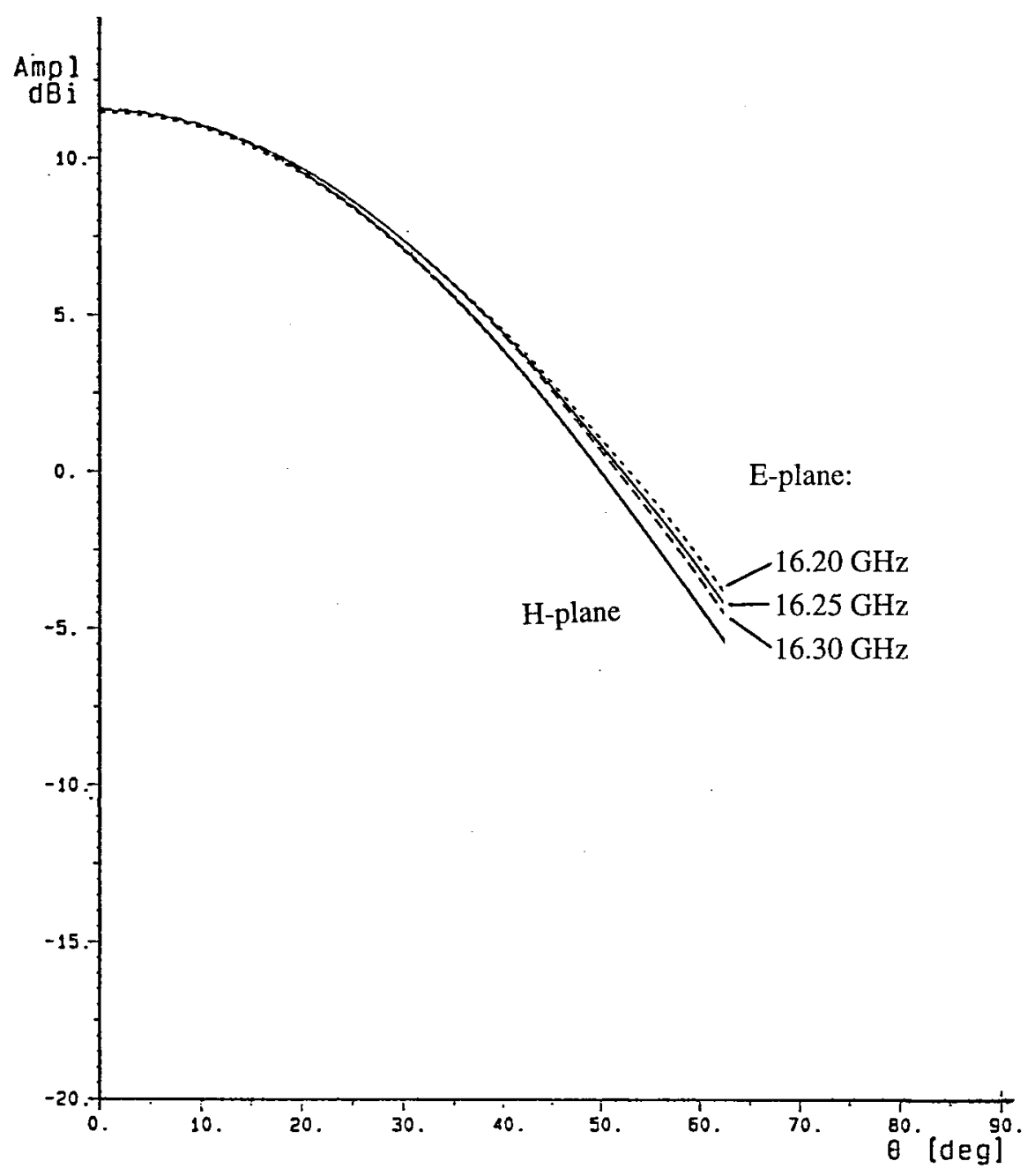

Fig. 5. Radiation pattern for the circular dual-mode horn, $E$ - and $H$-plane.

inversion using individual antenna patterns for each visibility sample (see [5] and [6]). The error bound $4.2 \mathrm{~K}$ is assessed by inspection of (19) in an actual case.

The phase reference corresponding to the far-field radiation pattern of the dual-mode horn is in the aperture; this is the case not only at the axis, but within the reconstructed field of view, with an accuracy better than $\pm 1.2^{\circ}$.

\section{C. $K_{u}$-Band SARad Block Diagram}

Temperature-stabilized, low-noise preamplifiers are placed right after the horn antennas, followed by phase-stable flexible microwave cables, to allow the placement of the antenna elements in all required positions.

A common LO (local oscillator) ensures channel coherence (see Fig. 6). The phase shifter between the LO and the mixer in channel 2 is used to compensate for any difference in electrical delay in the two receivers.

High isolation between the two channels is required, and the LO section is especially critical. Isolation at RF as well as IF frequencies are obtained by a combination of isolators and waveguide LO filters.

The power divider network, used to feed the analog correlators, consists of quadrature hybrids followed by inphase, two-way power dividers. The analog correlator is a fourquadrant analog multiplier followed by an integrator. An IF switch arrangement in front of the analog correlators enables the cross correlator to be used as self-correlators (identical to detectors) and, hereby, use the correlation radiometer as two total-power radiometers (the position of the IF switches in Fig. 6 corresponds to the situation in which the radiometer is used as a two-channel correlation radiometer). The calibration of the correlation radiometer is performed in a traditional way with the radiometer in total-power mode.

Offsets on the output of the correlators are reduced by phase switching of the local oscillator signals and proper demodulation after the correlator. The analog integration time $\tau$ is $8 \mathrm{msec}$, and the phase switching is done with $f_{s}=1 \mathrm{kHz}$, i.e. much faster than the integration time. The phase switching is only used when the radiometer is operated as a correlation radiometer. The final integration time is determined by the number of samples averaged in the computer used for data collection. Typically, $400 \mathrm{msec}$ integration is used, and the corresponding sensitivity is $\Delta T=0.1 \mathrm{~K}$.

Phase switching improves the stability of the radiometer considerably, but very small offsets still appears from the asymmetry in the \pm 1 demodulation and asymmetry in the duty 


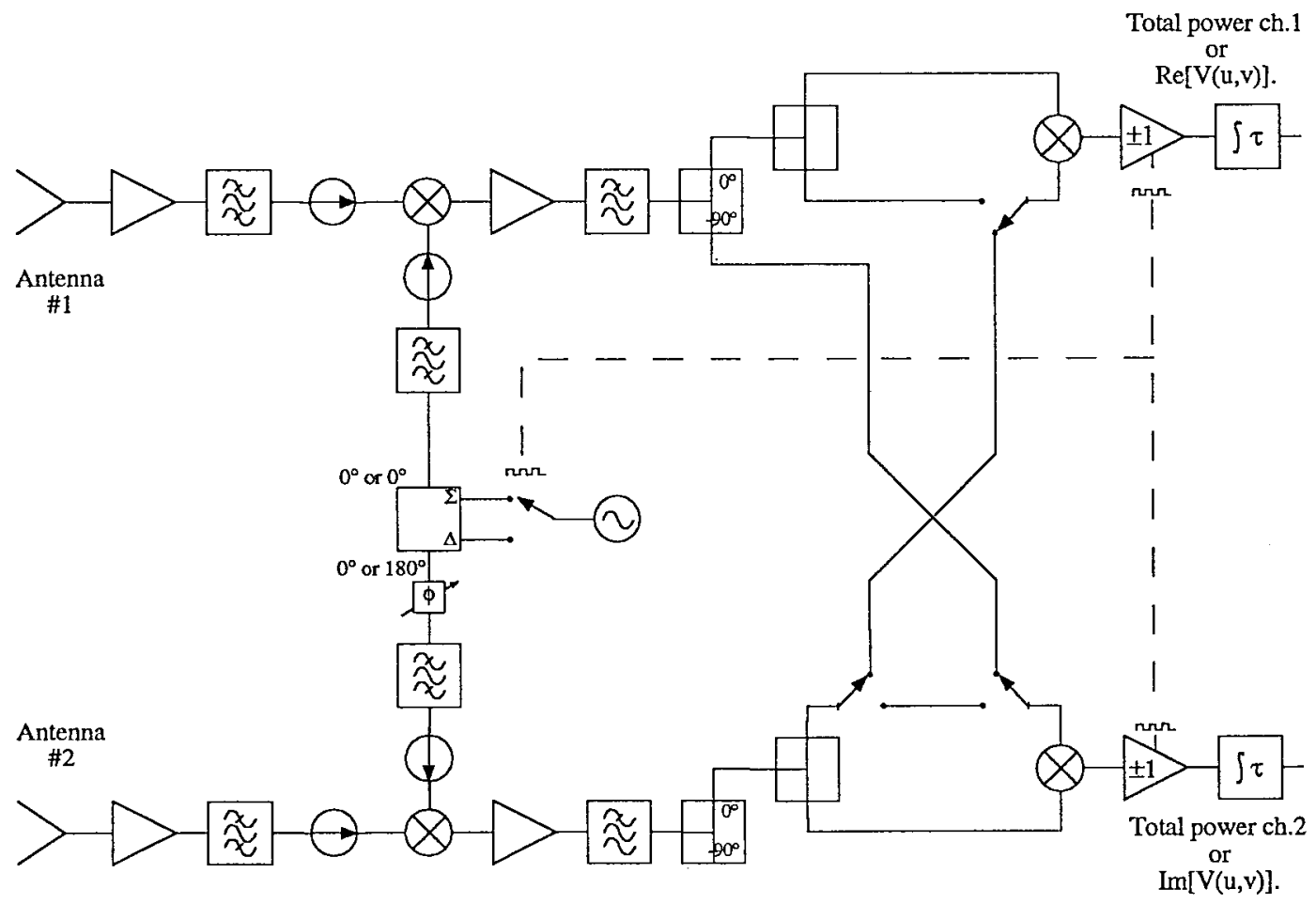

Fig. 6. Block diagram of the $K_{u}$-band correlation radiometer.

cycle of the phase switching signal. The A/D converter cannot be offset adjusted to the required level of accuracy either.

\section{Calibration}

Remaining offsets can be measured by injection of uncorrelated noise in both receivers. The brightness temperature from two independent absorbers in front of the antennas are measured with the two receivers. To evaluate this measurement, the result is compared with a measurement with two independent loads on the preamplifiers, and the result was identical. The method using absorbers is much faster when the uncorrelated noise calibration is done in between the measurement of visibility samples.

The calibration of the gain and noise figure is done with the IF switches in total-power radiometer mode, and two external reference temperatures are used. The sky is used as a cold reference temperature by pointing the antenna element at zenith. As a warm reference temperature, the antenna element is pointed at absorbers. At $K_{u}$-band frequencies, the zenith sky temperature is no longer insensitive to the water vapor content in the atmosphere, and even on a clear day, sky temperatures ranging $6-15 \mathrm{~K}$ can be expected for a $K_{u}$-band radiometer. A third well-known temperature can be applied with absorbers cooled by liquid nitrogen.

In order to achieve identical electrical delays in the two channels, the phase of the local oscillator signal in channel 2 is adjusted to maximize the $I$ output signal (minimize the $Q$ output) for equal, correlated input signals. These signals are generated by a common noise source $T_{2}$ and a Wilkinson power divider. $T_{2}$ must be different from the physical temperature of the power divider to obtain two correlated output signals of the power divider. The reason for this is the noise signal from the internal resistor in the power divider being $180^{\circ}$ out of phase on the output ports. When $T_{2}$ equals the temperature of the power divider, the effect of the two noise signals cancels in the correlation. $T_{2}$ is typically $400 \mathrm{~K}$, being strong enough for accurate phase adjustments, yet low enough to avoid saturation in the receivers. The accuracy of the phase adjustment is in the range of $\Delta \phi= \pm 5^{\circ}$.

\section{IMAGE RECONSTRUCTION}

As measurements with the SARad are performed in the spatial frequency domain, a Fourier transform is used to come back to the spatial domain (the image domain). The spatial frequencies are in most cases not placed on a rectangular grid, except for a T-shaped antenna, and a traditional inverse FFT is therefore not suitable.

The image reconstruction is carried out by calculating two different maps, the dusty map and what is called the final map. The inverse transform required in (19) is in general derived in [7] and [8], and the dusty map is

$$
\begin{aligned}
y \boldsymbol{p}(\xi, \eta)= & d u d v \sum_{(u, v) \in L_{e}} \hat{B}\left(\frac{u}{\Delta u}\right) \hat{B}\left(\frac{v}{\Delta v}\right) \\
& \times \frac{V_{e}^{\prime}(u, v)}{\rho(u, v)} W(u, v) e^{-j 2 \pi(u \xi+v \eta)}
\end{aligned}
$$

where

$$
\hat{B}(v) \quad F\{B(\zeta)\} \text { is an interpolation function. }
$$

$L_{e}$ is the list of measured spatial frequencies; $W(u, v)$ is a Hamming weighting function; and $\rho(u, v)$ is accounting for redundant samples of the visibility function [in our case, see 
Fig. 3(b), substantial oversampling takes place near the center of the $u-v$ plane]

$$
\rho(u, v)=\sum_{\left(u^{\prime}, v^{\prime}\right) \in L_{e}} \hat{B}^{2}\left(\frac{u-u^{\prime}}{d u}\right) \hat{B}^{2}\left(\frac{v-v^{\prime}}{d v}\right) .
$$

The dusty map (20) is calculated on a rectangular $(\xi, \eta)$ grid, denoted $G$ with $(n \times n)$ number of points.

The so-called final map $\phi_{i+1} \boldsymbol{p}$ is calculated as

$$
\phi_{0 \boldsymbol{p}}=P_{E} y \boldsymbol{p} \quad \text { and } \quad \phi_{\boldsymbol{i}+1} \boldsymbol{p}=\phi_{i \boldsymbol{p}}+\omega_{i} r_{i} \boldsymbol{p} \quad\left(\boldsymbol{p} \in G_{1}\right)
$$

where $P_{E}$ is the circular restriction on the grid with a diameter of $n-2, G_{1}$ is a grid $(2 n \times 2 n)$ twice as large as $G$, the residue map is given by

$$
r_{i} \boldsymbol{p}=\phi_{0} \boldsymbol{p}-P_{E} A^{*} A \phi_{i} \boldsymbol{p} \quad\left(\boldsymbol{p} \in G_{1}\right)
$$

and the relaxation parameter is given by

$$
\omega_{i}=\frac{\sum_{\boldsymbol{p} \in G} r_{i} \boldsymbol{p}_{i}^{*} \boldsymbol{p}}{\sum_{\boldsymbol{p} \in G} r_{i} \boldsymbol{p}\left(P_{E} A^{*} A r_{i} \boldsymbol{p}\right)} .
$$

The action of the operator $A^{*} A$ on a map (used both on the residue map and in the relaxation parameter) is calculated as the discrete convolution between the respective map and the dusty beam, using rectangular FFT, where the dusty beam contains information about the regularization errors of the visibility function (the problem of coming from the nonregular distribution of the visibility samples to a distribution on a rectangular grid). The processing of measurements on a nonrectangular grid to a rectangular grid is straightforward in the case of a visibility function without errors. In any real situation, both additive as well as multiplicative errors (offset and gain errors, phase and amplitude) are present. The correction for these errors are attempted in the calibration. However, using the image reconstruction formulation $A^{*} A$ from [7] and [8], the brightness temperature image is reconstructed without additional errors from the interpolation, even in the case where the calibration has not completely removed the instrument errors.

Equations (20) and (22) are in both cases taking care of the inverse Fourier transform of the visibility function in (19) $\left(F^{-1}\left\{V_{e}^{\prime}(u, v)\right\}\right)$, and the antenna gain corrections is in all cases applied afterwards, as described in (19).

Finally a ground projection is performed to obtain a true image of the measured brightness temperature distribution as a function of ground coordinates $\left(x_{G}, y_{G}\right)$.

$$
\begin{aligned}
& x_{G}=h \frac{\xi}{\sqrt{1-\xi^{2}-\eta^{2}}} \\
& y_{G}=h \frac{\eta}{\sqrt{1-\xi^{2}-\eta^{2}}} .
\end{aligned}
$$

\section{SARad IMAGES}

Three images are presented in the following. They are measured with the TUD $K_{u}$-band SARad system in its nearfield configuration, the case where it is relatively straightforward to make complex and well-controlled targets at a reasonable distance from the antenna. Metal targets reflecting
TABLE I

SPECIFICATIONS FoR the TUD $K_{u}$-BAND Two-Channel SARad System

\begin{tabular}{l|c}
\hline Parameter: & Specification: \\
\hline Frequency & $16.25 \mathrm{GHz}$ \\
\hline System bandwidth & $100 \mathrm{MHz}$ \\
\hline Noise figure & $3 \mathrm{~dB}$ \\
\hline "Altitude" & $1.354 \mathrm{~m}$ \\
\hline Aperture & $32 \lambda(58 \mathrm{~cm})$ \\
\hline Synthetic beamwidth (center) & $2.2^{\circ}(5.2 \mathrm{~cm})$ \\
\hline Antenna element type & Circular dual mode horn $(1.5 \lambda)$ \\
\hline Number of visibility samples & 1065 \\
\hline Spatial frequency sampling & $1.57 \lambda$ \\
\hline Field of view & $\pm 18.5^{\circ}( \pm 45 \mathrm{~cm})$ \\
\hline Image size & $18 * 18$ pixels \\
\hline
\end{tabular}

the sky temperature (low brightness temperature) against a warm uniform background (absorbers) have been successfully imaged. The main specifications are shown in Table I.

The target in Fig. 7 is a background of absorbers with a brightness temperature equal to the physical temperature (288 $\mathrm{K})$ and with three metal letters spelling "EMI" placed on top of the absorbers. All measurements are taken outdoors, so the metal letters are reflecting the cold sky temperature. The letters are made out of metal sticks with a width of $5 \mathrm{~cm}$ (seen on the photo in the upper part of Fig. 7). The resolution is of the same order as the width of the metal sticks: $5.2-\mathrm{cm}$ theoretical resolution at the center of the image using a Hamming weighting function.

Two metal sticks (3-cm wide and $1.2-\mathrm{m}$ long) were placed on the absorbers to keep them fixed on a rather windy day. The sticks are seen in the final map as two horizontal lines above and below the "EMI." The brightness temperature is not as cold as for the metal letters due to the small width (these long sticks are not seen on the photo).

The resolution can be checked toward the edge of the final map by observing the dot on the "I" (in "EMI"), which has the same size as the theoretical resolution. The reconstructed image is $90 \times 90 \mathrm{~cm}$. Aliasing is removed by placing absorbers outside the $90 \times 90-\mathrm{cm}$ area, solving the integral in (17). A part of the antenna mounting structure was not covered correctly by absorbers for this particular measurement. The assumption of $288 \mathrm{~K}$ is therefore not correct for this area, and the structure is seen as the yellow strip at $x=+0.2 \mathrm{~m}$ (aliased image). This problem is not present in the following measurements. The image still contains a number of artifacts, and they will be commented in the discussion of the following images.

The target in Fig. 8 is a metal plate reflecting the cold sky temperature. The metal plate placed at the center is $22.5 \times 28 \mathrm{~cm}(5 \times 6$ pixels $)$. The background is absorbers with a brightness temperature of $290 \mathrm{~K}$. A fairly simple target of two well-defined brightness temperature levels is a useful scene to test calibration properties. 

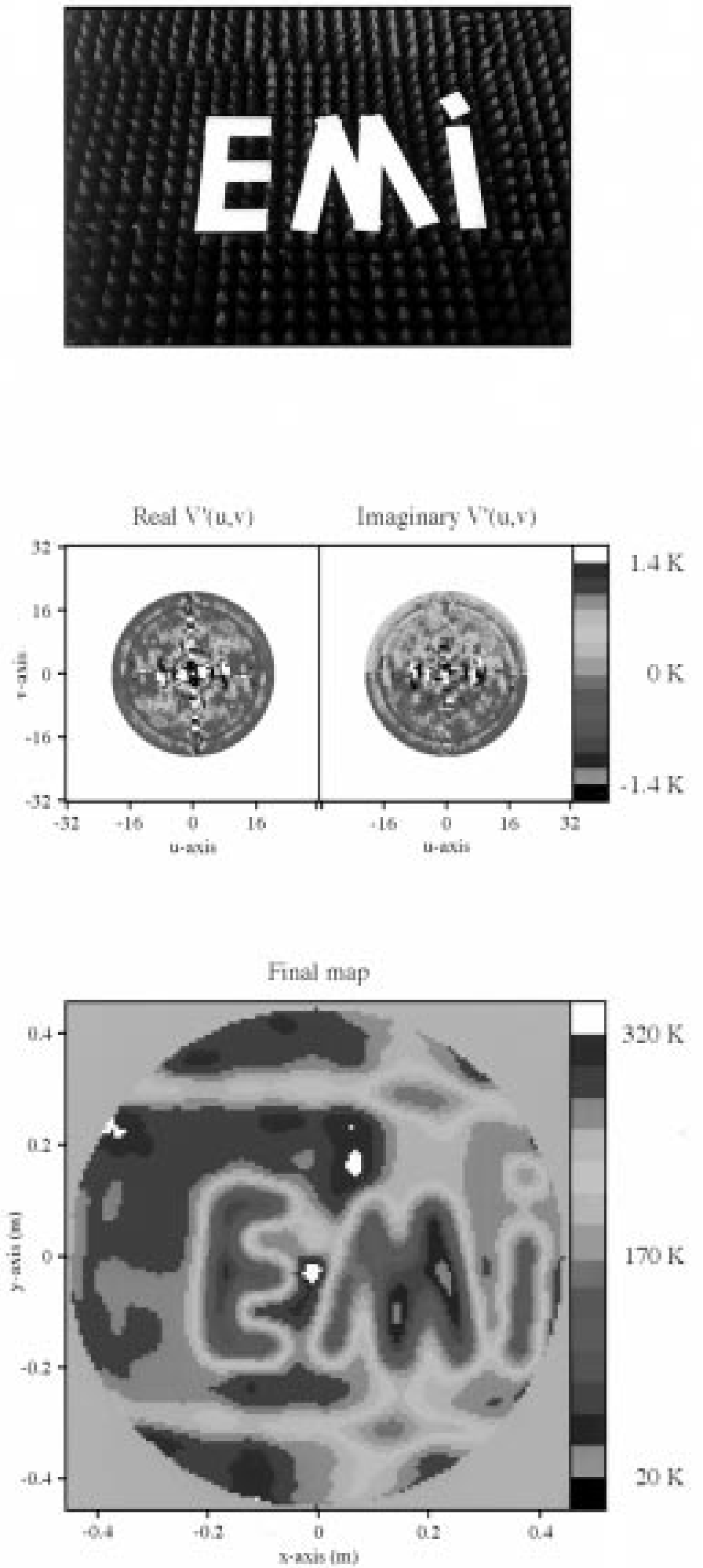

Fig. 7. Brightness temperature image of metal letters spelling "EMI."

The images from the TUD SARad system have previously suffered from a large peak at the center of the image, where the brightness temperature would rise up to $100 \mathrm{~K}$ (see [9]). It is argued that a constant offset on all visibility samples were giving this peak because all offset errors tend to add up in the Fourier transform when the image is reconstructed.
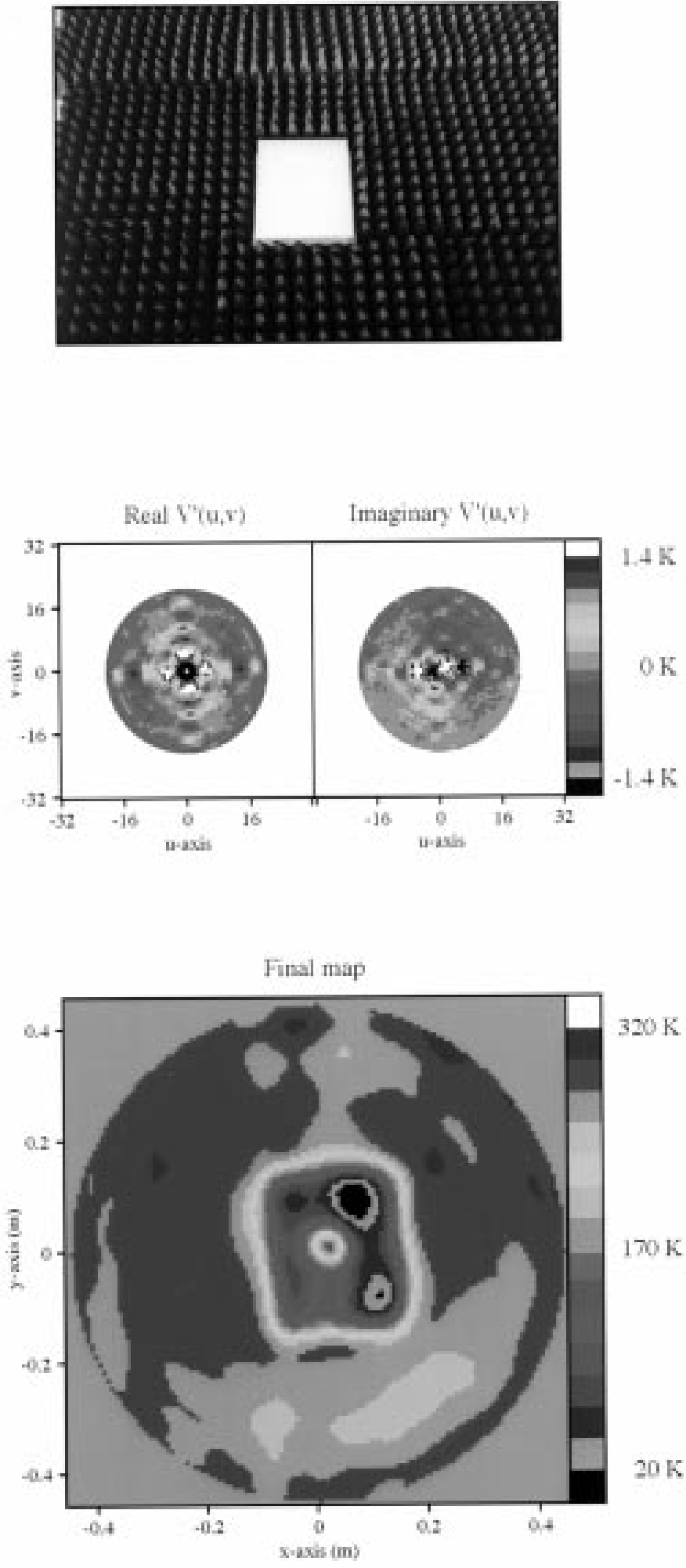

Fig. 8. Brightness temperature image of $5 \times 6$-pixel metal plate reflecting the sky temperature.

Even small offsets of a few tenths of a $\mathrm{K}$ will add up to this kind of artifact, when the error is constant for all visibility samples.

Phase switching the local oscillator signals and proper demodulation after the correlator will cancel these offsets. The peak is reduced, compared to the previous results, but 

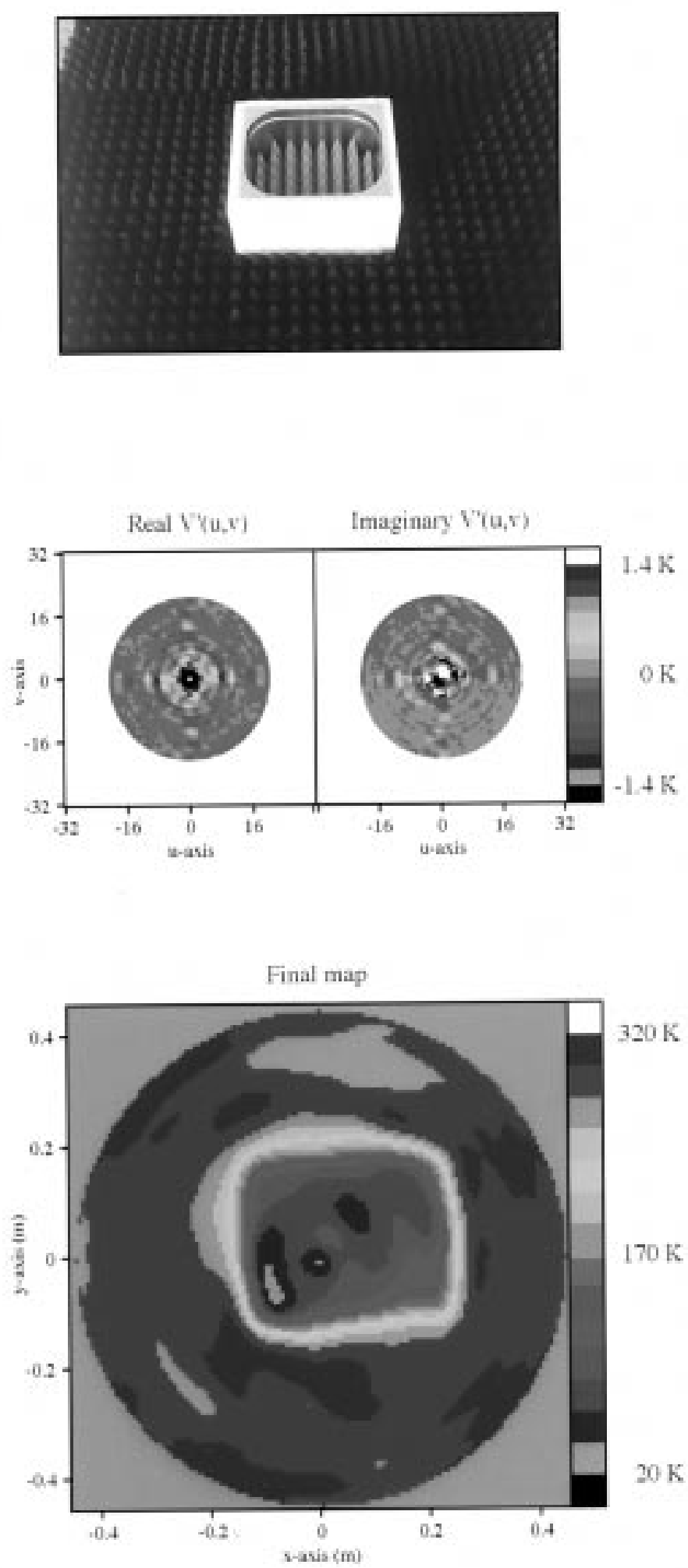

$320 \mathrm{~K}$

Fig. 9. Brightness temperature image of $7.3 \times 6.6$-pixel liquid nitrogen cooled absorber.

not fully eliminated, as evident in Fig. 8. The reason for the remaining peak was found to be reflections in the metal plate of warmer temperatures from the near-field antenna structure placed above the target in an altitude of $1.35 \mathrm{~m}$. This result was confirmed with the measurement shown in Fig. 9.
The target in Fig. 9 is not based on reflection, but purely on radiation from absorbers. The background is absorbers with a brightness temperature of $300 \mathrm{~K}$. A thermal box is placed at the center with absorbers cooled by liquid nitrogen, giving a brightness temperature of $77 \mathrm{~K}$. The size of the cooled absorbers is $33 \times 30 \mathrm{~cm}(7.3 \times 6.6$ pixels $)$. The target is still based on two well-defined brightness temperature levels but no reflection mechanism.

The cooled absorbers are seen on the photo in the upper part of Fig. 9 (blue absorbers). The cooled absorbers are placed in a stainless steel box, and the box is isolated with polystyrene foam. A radome is placed on top of the box (not shown in the photo) to keep the nitrogen liquid for several hours (the measurement takes about $2 \mathrm{~h}$ ). The radome is transparent to the microwave radiation, while the isolation is still high enough to avoid condensation on the inside of the radome. The peak is no longer present in the final map shown in Fig. 9.

Other errors, apart from the peak, are seen in all three images, in the absorber area as well as in the cold area. Fig. 9 reveals quite a large variation in the brightness temperature, both in the area with warm absorbers and in the area with absorbers cooled by liquid nitrogen. The peak-to-peak variation is as much as $70 \mathrm{~K}$ in the cold area and $30 \mathrm{~K}$ in the warm area.

\section{CONCLUSION}

Using simple hardware (only two radiometer channels) it has been possible to demonstrate the thinned array radiometer concept with aperture synthesis in both directions and experience some of the problems associated with this technique.

The imagery of complicated targets, at a practical short distance, have been acquired by using a focused antenna technique. It has been shown that, by proper implementation of this technique, the normal far-field image reconstruction algorithms are still valid.

Two-dimensional image reconstruction has been carried out with success, and the resolution compares well with theoretical predictions.

A series of measurements has shown that offset problems may seriously degrade the correlation measurements, but the inclusion of phase switching greatly reduces errors. The measurements also showed problems with reflections in the antenna structure when relying on targets reflecting the cold sky into the radiometer antennas. This was clarified using scenes consisting entirely of absorbers (pure radiation mechanism) having different physical temperatures.

The demonstration model can be used to evaluate future spaceborne systems, while they are still in their design phase. For example, the robustness of the image reconstruction algorithm can be investigated by introducing typical errors on the visibility samples (including missing samples caused by failing radiometer channels). Such work is in progress.

The demonstration model also has its limitations, which must be observed. We have tried to investigate calibration topics. However, measurement errors tend to add in images from a two-channel radiometer, where all samples are taken by the same two receivers in a way not realistic concerning multichannel correlation radiometers. 


\section{ACKNOWLEDGMENT}

Dr. R. Davidheiser of TRW has contributed to the work through many fruitful discussions during the initial phases.

\section{REFERENCES}

[1] A. R. Thompson, J. M. Moran, and G. W. Swenson, Interferometry and Synthesis in Radio Astronomy. New York: Wiley, 1986.

[2] C. S. Ruf, C. T. Swift, A. B. Tanner, and D. M. Le Vine, "Interferometric synthetic aperture microwave radiometry for the remote sensing of the earth," IEEE Trans. Geosci. Remote Sensing, vol. 26, pp. 597-611, Sept. 1988.

[3] J. M. Goutoule, U. Kraft, and M. Martin-Neira, "MIRAS: Preliminary concept of a two-dimensional $L$-band aperture synthesis radiometer," in Microwave Radiometry and Remote Sensing of the Environment, D. Solemini, Ed. Amsterdam, The Netherlands: VSP, 1995.

[4] B. Laursen and N. Skou, "A spaceborne synthetic aperture radiometer simulated by the TUD demonstration model," IGARSS'94, pp. 1314-1316.

[5] A. B. Tanner and C. T. Swift, "Calibration of a synthetic aperture radiometer," IEEE Trans. Geosci. Remote Sensing, vol. 31, pp. 257-267, Jan. 1993.

[6] J. Bara, A Camps, I. Corbella, and F. Torres, "Bi-dimensional discrete formulation for aperture synthesis radiometers," ESTEC, Noordwijk, The Netherlands, Contract 9777/92/NL/PB Final Rep., Jan. 1996.

[7] A. Lannes, E. Anterrieu, and K. Bouyoucef, "Fourier interpolation and reconstruction via Shannontype techniques. I. Regularization principle," J. Modern Opt., vol. 41, no. 8, pp. 1537-1574, 1994.

[8] A. Lannes, E. Anterrieu, and K. Bouyoucef, "Fourier interpolation and reconstruction via Shannontype techniques. II. Technical development and applications," J. Modern Opt., vol. 43, no. 1, pp. 105-138, 1996.

[9] B. Laursen, H. M. Pedersen, and N. Skou, "The TUD synthetic aperture radiometer demonstration model," in Microwave Radiometry and Remote Sensing of the Environment, D. Solemini, Ed. Amsterdam, The Netherlands: VSP, 1995.

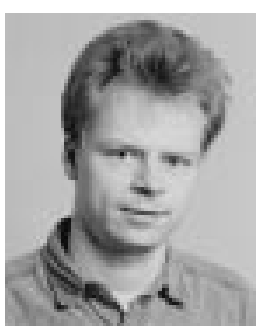

Brian Laursen (M'97) received the M.Sc. degree from the Technical University of Denmark, Lyngby, in 1992. He is currently pursuing a Ph.D. degree from the Department of Electromagnetic Systems, Technical University of Denmark.

His research has focused on microwave radiometer systems. Correlation radiometry has especially been his main interest, using the correlation technique in synthetic aperture radiometry as well as polarimetric radiometry.

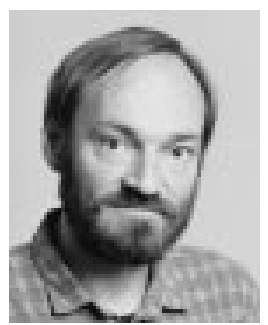

Niels Skou (S'78-M'79-SM'96) received the M.Sc., Ph.D., and D.Sc. degrees from the Technical University of Denmark, Lyngby, in 1972, 1981, and 1990, respectively.

$\mathrm{He}$ is currently an Associate Professor in the Department of Electromagnetic Systems, Technical University of Denmark. His research has been directed toward microwave remote sensing systems. After working for three years with the development of radar systems for measuring the ice sheets in Greenland and Antarctica, his interest turned toward microwave radiometry. From 1975 to 1979 , he developed a scanning, multifrequency, airborne radiometer system. After that, his subjects were radiometer measurements of sea ice and oil pollution on the sea, spaceborne radiometer systems, and development of new systems for specific purposes. In the mid-1980's, his interest turned back to active instruments and he became engaged in the development of airborne synthetic aperture radar systems. However, some activity within microwave radiometry has continued, mainly within the relatively new areas of synthetic aperture radiometry and also polarimetric radiometry. 